\title{
Medical residents' feedback on needs and acquired skills following a short course on cross-cultural competence
}

\author{
Alicia Bardet ${ }^{1}$, Alexander R. Green ${ }^{2}$, Sophie Paroz ${ }^{3}$, Pascal Singy ${ }^{4}$, Paul Vaucher ${ }^{3}$, Patrick Bodenmann ${ }^{3}$ \\ ${ }^{1}$ Faculty of Biology and Medicine, University of Lausanne, Switzerland \\ ${ }^{2}$ The Disparities Solutions Centre, Massachusetts General Hospital, Harvard Medical School, USA \\ ${ }^{3}$ Department of Ambulatory Care and Community Medicine, University of Lausanne, Switzerland \\ ${ }^{4}$ Department of Psychiatry, University of Lausanne, Switzerland \\ Correspondence: Patrick Bodenmann, Bugnon 44, CH-1011 Lausanne, Switzerland. Tel. +41 213144937. \\ E-mail: patrick.bodenmann@hospvd.ch
}

\begin{abstract}
Objectives: The purpose of this study is to assess short and long term changes in knowledge, attitudes, and skills among medical residents following a short course on cultural competency and to explore their perspectives on the experience.

Methods: Eighteen medical residents went through a short training programme comprised of two seminars lasting 30' and 60' respectively over two days. Three months later, we conducted three focus groups, with 17 residents to explore their thoughts, perspectives and feedback about the course. To measure changes over time, we carried out a quantitative sequential survey before the seminars, three days after, and three months later using the Multicultural Assessment Questionnaire.
\end{abstract}

Results: Residents expressed a wide variety of perspectives on the main themes related to the content of the training culture, trialogue, stereotypes, status, epidemiology, history and geopolitics - and related to its organization - relevance, volume, timing, target audience, training tools, and working material. Using the MAQ, we observed a higher global performance score $(n=16)$ at three days (median=38) compared to results before the training (median=33) revealing a median difference of 5.5 points $(\mathrm{z}=2.4, \mathrm{p}=0.015)$. This difference was still present at three months $(\Delta=4.5$, $\mathrm{z}=2.4, \mathrm{p}=0.018)$, mainly due to knowledge acquisition $(\Delta=3)$ rather than attitudes $(\Delta=0)$ or skills $(\Delta=1)$.

Conclusions: Cross-cultural competence training not only brings awareness of multicultural issues but also helps participants understand their own cultures, perception of others and preconceived ideas. Physicians' education should however also focus on improving implementation of acquired knowledge in cross-cultural competence.

Keywords: Education, medical, graduate, cultural competency, emigrants and immigrants, primary health care

\section{Introduction}

Providing medical care to immigrant patients is a major challenge for health care professionals. Physicians, in particular, have to deal with significant linguistic and cultural differences as well as differences in patterns of disease for patients from countries with diverse epidemiological profiles. Numerous studies have shown that to reduce potential inequalities and barriers to care for immigrants it is important for healthcare personnel and physicians to be trained in cross-cultural competence (CCC).,

In medical care, CCC is the ability to provide quality care to patients from different socio-cultural backgrounds.
More specifically, cardinal aspects of CCC include the ability to manage language barriers, communication styles, mistrust and prejudice, family dynamics, customs and spirituality, and sexual and gender issues. CCC depends also on demonstrating empathy, curiosity, and respect, key factors for effective patient care in a multicultural context. ${ }^{3}$ In the teaching and learning of CCC, three major domains are described and systematically studied by experts in the field: ${ }^{4-9}$ knowledge, attitudes and skills. The first domain knowledge - may comprise, for example, information related to migration status (permits, social instability), 
epidemiological knowledge and/or medical anthropology (influence of culture on the representation of symptoms and illness).

The second domain is attitudes: self-awareness of one's own socio-cultural background, and the potential for stereotyping. Finally, the skills domain describes the ability to communicate effectively across cultures (including but not limited to use of interpreter services), to build trusting relationships, and to adapt care to meet the patient's unique social and cultural perspective. Competence in these three domains enables patients to receive good medical care despite differences both in language, culture. Other factors such as gender, age, social status, disability, and sexual orientation are also integrated into the concept of CCC. ${ }^{10,11}$ In summary, to care for immigrant patients it is important to explore beliefs concerning illness and treatment, ${ }^{12}$ to assess cross-cultural issues, ${ }^{3}$ to address language and literacy ${ }^{13}$ and determine the social context. ${ }^{14}$

There is a wide range of studies on the effects of cultural competency training of various lengths, that target different clinical disciplines, emphasize different core concepts, and measure different outcomes. ${ }^{15,16}$ Most of these have shown improvement in knowledge, attitude and/or skills. A few have shown improvements in patient outcomes. However, from this diversity of studies, no clear pattern emerges as to which approaches are most successful. In Switzerland, a country that recognizes four official languages (German, French, Italian and Romanche), $21.2 \%$ of the population $(1,714,000$ people) is of foreign origin. In the city of Lausanne, in the French-speaking part of the country, immigrants account for $38.8 \%$ of its 180,000 inhabitants. ${ }^{17,18}$ In this diverse setting, the impact of a training program for internal medicine residents has not yet been assessed. Our objective is therefore to assess short and long term changes in knowledge, attitudes, and skills following a short course on cultural competency.

\section{Methods}

\section{Participants}

In the "Policlinique Médicale Universitaire" (PMU) of Lausanne there are 33 internal physicians in training and every six months there is a rotation of nearly half the team. We surveyed all of the PMU's 18 new primary care residents (their characteristics are described in Table 2) during the compulsory first day of training, with a voluntary paperand-pencil survey.

\section{Settings}

The PMU is a major centre for academic ambulatory internal medicine, and plays an important role in immigrant healthcare for the region. In 2009, approximately 15,000 emergency visits and 15,000 outpatient visits were recorded at this institution. Each year around 1,700 patients considered "migrants in transition" (requesting asylum, denied asylum or without documentation) receive medical care at PMU, and around 5,000 hours of contact take place with a trained staff interpreter present.

\section{Teaching program}

Since 2005, Lausanne's PMU has provided in-service programs in CCC for resident physicians, and courses were initiated for medical students in 2007. For this study, we evaluated the resident course taught two times a year. It consists of two seminars given over a two-day period. Details of both seminars are given in Table 1. The first course focuses on the practical, financial and managerial approaches to immigrant populations. In the second phase, the training is centred on more theoretical aspects of CCC, with information on culture in general, stereotypes and effective use of interpreter services (trialogue). Videos are used to illustrate the challenges of working with an interpreter.

\section{Qualitative methods}

Seventeen of the 18 residents participated in the qualitative part of the study (one was not available). We conducted three focus groups using participant interaction to explore residents' viewpoints and experiences. ${ }^{13,19}$ We initially planned to form groups based on residents' gender, years of medical experience, language and country of origin. However, due to challenges faced with the complexity of schedules, we formed the groups according to the residents' availability. We inquired about several key topics in the focus groups: 1) how interesting the training was, 2) utility of the training, 3) training placement in the faculty syllabus, 4) expectations, 5) introducing the concept of culture into the consultation, 6) risk of stereotyping, and 7) working with an interpreter. Since CCC training is a fairly recent addition to a medical curriculum in Switzerland, we included qualitative methodology in order to better understand the residents' perspectives on this topic and the trainings themselves. Each focus group lasted approximately one hour and was directed by the first and last author of this paper (the latter being one of the CCC clinical trainers). The discussions were recorded and transcribed carefully and then put through a thorough qualitative content analysis to establish emerging themes. Technical support during the focus group was provided by a separate person than the one who moderated the discussion. This consisted of preparing the material, managing the audio recording, and noting all non-verbalised important interactions. A table of codes with major themes and minor themes was established by a transdisciplinary group of analysts (five participants), based on a random reading after the transcription, following the predefined categories of knowledge, attitude and skills.

\section{Quantitative approach}

A pre-post intervention comparative design was used to compare CCC before, just after, and three months after the cultural competency training. 
Table 1. Description of short training programme

\begin{tabular}{|c|c|c|}
\hline Characteristics & Seminar 1 & Seminar 2 \\
\hline Global objectives & $\begin{array}{l}\text { Familiarise residents with practical, financial and managerial } \\
\text { approaches to immigrant populations. }\end{array}$ & $\begin{array}{l}\text { Introduce cross-cultural competence and illustrate the } \\
\text { importance of general culture knowledge and recognising } \\
\text { stigmatisation when taking care of a patient. Instruct how to } \\
\text { overcome language barriers using a third person for } \\
\text { translations (trialogue). }\end{array}$ \\
\hline Specific objectives & $\begin{array}{l}\text { Residents are to: } \\
\text { Define different migration status and underlying health care } \\
\text { specific legal conditions. } \\
\text { Identify correct administrative procedures for each type of } \\
\text { migrant. } \\
\text { Recognise financial difficulties to manage health costs and } \\
\text { offer existing administration solutions. }\end{array}$ & $\begin{array}{l}\text { Residents are to: } \\
\text { Define and illustrate the importance of cross-cultural } \\
\text { competences. } \\
\text { Conceive relations between patients' cultural background, } \\
\text { epidemiological data, and patients' perception of disease or } \\
\text { health care. } \\
\text { Plan and handle a three-way exchange with an interpreter } \\
\text { (trialogue). }\end{array}$ \\
\hline Duration & $30 \mathrm{~min}$ & $60 \mathrm{~min}$ \\
\hline Number of participants & 18 residents or chief residents & 18 residents or chief residents \\
\hline Supervisors and lecturers & $\begin{array}{l}\text { A senior lecturer and a chief resident both with extended } \\
\text { experience in working with vulnerable population. }\end{array}$ & $\begin{array}{l}\text { A senior lecturer and a chief resident both with extended } \\
\text { experience in working with vulnerable population. }\end{array}$ \\
\hline Educational strategies & Cognitive approach & Cognitive approach integrating constructive principles \\
\hline Teaching methods & Lecture with visual support (slides and videos) & $\begin{array}{l}\text { Lecture with visual support followed by three case studies } \\
\text { using video and opening the discussion on what is positive } \\
\text { and what can be improved. }\end{array}$ \\
\hline Mental process & $\begin{array}{l}\text { Passive learning: elaboration, organisation, and procedurali- } \\
\text { sation. }\end{array}$ & $\begin{array}{l}\text { Passive and active learning: organisation, proceduralisation, } \\
\text { composition, generalisation, and discrimination. }\end{array}$ \\
\hline \multicolumn{3}{|l|}{ Knowledge } \\
\hline Type & Declarative and procedural knowledge & Procedural and conditional knowledge \\
\hline Source & Legal basis, research, and experience & Research and experience \\
\hline Support documents & $\begin{array}{l}\text { Power point slides given after the course. } \\
\text { Summary flyers for practical use. }\end{array}$ & Power point slides given after the course. \\
\hline
\end{tabular}

\section{Measure of performance}

Through a literature review, we searched for tools to assess CCC teaching and selected a standardized and validated questionnaire for the quantitative part of the study. We chose the "Multicultural Assessment Questionnaire" (MAQ) from Culhane-Pera and colleagues. ${ }^{20}$ because it is composed of few questions, focuses on residents, and is divided into three domains of CCC. It consists of sixteen questions, of which six concern knowledge, four attitude, and six skills. This instrument has been considered as the reference standard for measuring CCC in the medical field ${ }^{21}$ and is recommended by various systematic literature reviews regarding tools for the evaluation of CCC training. ${ }^{15,22}$ The original English version was translated to French by professional translators. We then back-translated the French version to English to verify consistency with the original version. The response variable was a scale of selfperceived preparedness to care for immigrant patients; we inquired about participants' knowledge, attitude and skills in caring for these patients on a 5 -point scale $(0=$ none, $4=$ excellent).

\section{Data collection}

Data collection was carried out following a pre-post study design. The physicians completed the questionnaire for the first time before the CCC training began (D-1). The questionnaire was given for the second time on 6 April 2010, although participants were not yet involved in clinical activities $(\mathrm{D}+3)$. The questionnaire was completed for a third time, after three months of clinical practice, by the primary care physicians $(\mathrm{D}+90)$. The study was compliant with ethical regulations. The study protocol was reviewed and accepted by the official state ethics committee of Vaud for biomedical studies in December 2009.

\section{Data analysis}

MAQ sub-scores were obtained by summing all Likert scale scores (0-4) within each category: knowledge (0-24 points), attitude (0-16 points) and skills (0-24 points). Global index, our principle outcome, was obtained by summing the three sub-scores (0-64 points). Medians of differences were used instead of difference in means. Significant level of median difference different from 0 was set at $\mathrm{p}=0.05$ using Wilcoxon's signed-rank test for sub-scores.

\section{Results}

\section{Qualitative dimension}

Using the data collected during the three focus group interviews (including five, nine, and three participants), three authors classified participants' comments and easily agreed on structure and labelling of categorisation. By classifying acquired skills and expectations according to the axes of knowledge, attitude and skills, different categories emerged from the interviews depending on the content (A) and organization (B) of the training course. 


\section{Content of the training course}

We analyzed the data from the focus group discussions according to the following categories of content in the teaching program: Culture / Trialogue / Stereotypes / Status / Epidemiology / History and geopolitics.

\section{Culture}

It is often difficult for physicians to explore a patient's culture during their consultations, particularly so for those providing emergency care as they generally see the patient only once and generally do not use trained interpreters. As a result, uncomfortable and frustrating situations can develop for both physician and patient.

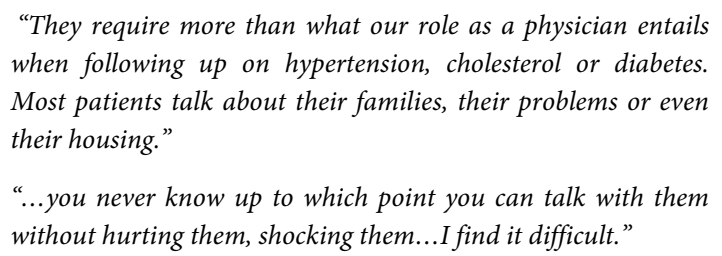

Primary care residents wish to acquire a range of knowledge regarding different cultural aspects. Some seek a sort of magic formula "What to do with patient X; what to avoid with patient $X$ ?" which is a simplified version of culture that is inapplicable in clinical practice. This also shows that physicians need to feel safe in these complex situations.

\section{Stereotypes}

There is a major risk of stereotyping in this type of consultation. The best way of addressing this risk, according to the physicians, is to take the necessary time with the patient, arrange for a time to meet the patient again, and know the patient's "life story". On the other hand, for some participants, having stereotypes and being aware of them enables them to be attentive to some specific points and to go beyond such limitations.

It is difficult however not to trivialize these factors particularly when confronted with recurring requests and issues that are more frequently encountered in certain communities. "Boli-boli syndrome" is a prime example. In Serbo-Croat "Boli" means "pain" and residents feel that patients coming from the Balkans seem to describe another new pain at each consultation, leading to frustration and difficulties understanding the underlying cause. According to these residents it is always difficult to validate each complaint without any unhelpful afterthoughts. However, as one physician states:

"We have more stereotypes with diagnostics than with culture."

This shows that it is necessary to be attentive to a broad range of matters and not merely to focus on purely ethnicity-related, preconceived ideas.

\section{Trialogue}

The majority of the residents retained important knowledge from the three-way conversation training, such as asking short questions, looking at the patient, spending some time with the interpreter, reminding each other about medical confidentiality, etc. They noted that these few clues help them in their consultations. However, they also express the difficulties encountered in practice with some interpreters, and - more importantly - the difficulties of even obtaining one.

“...The first barrier... is language... and when the language barrier is overcome, it represents such an effort that we may not pay attention to the cultural level ..."

\section{Training course organization}

Training course organization was analyzed according to the following categories: Relevance / Volume / Timing / Target audience / Training tools / Working material.

\section{Relevance}

Most primary care physicians were glad to have attended this training course and found it beneficial. They were notably pleased to learn about the different types of resident's permits provided by the migration office, of the way of life of immigrants in Switzerland, and also, from an administrative standpoint, about healthcare provided to immigrants in the Lausanne area, all of which helps them in their practice. The participants described their interest and the pleasure of being in contact with these types of patients, and what inherent cultural aspects can bring to their consultation.

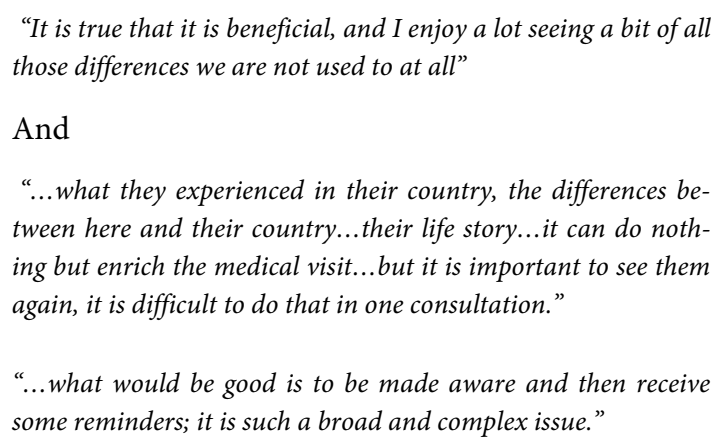

And

"...what they experienced in their country, the differences between here and their country...their life story...it can do nothing but enrich the medical visit...but it is important to see them again, it is difficult to do that in one consultation."

"...what would be good is to be made aware and then receive some reminders; it is such a broad and complex issue."

\section{Volume}

Physicians reported difficulty applying the principles of CCC due to the lack of time they were given to become comfortable with them. They express their need for more training time in their schedule, for the material to be repeated regularly in different forms, and to include group discussions in their training on quality of care for populations from different cultural backgrounds.

\section{Timing}

Many of the physicians had several suggestions regarding the program's schedule. Most groups felt it was not ideal to receive most of the information during the orientation day, upon their arrival. In addition to the main course physicians would also appreciate some one-off refresher courses. 
"...It is so important for our job that it deserves a specifically dedicated moment."

Some also want the possibility to have some practical experience before the course in order to have been confronted with real situations. In this way, they can better internalize the information received and have questions to ask during the training course.

"What I found beneficial is how other cultures see us as representative of a given nationality"... "It is amazing to see the recent historical influence on the perception of nationalities and culture."

\section{Target audience}

When asked about the best moment in their career to attend this training course the opinions of the primary care residents were divided. In general, most believed that it is essential in the post-graduate (residency) context. Conversely, could it be useful to teach this course to undergraduates? This question opened a debate, with some participants tending to feel that this would be too early in the university syllabus because it would serve no purpose if students had not yet been directly faced with the situation in question. Students have other concepts to learn at that time, and they may not consider it as important without an exam at the end. Other participants said that students must be made aware of it as early as possible, as some ideas can be incorporated into general knowledge acquisition and others into community medicine.

"There are some kinds of dogmas which are truly very local. Opening up our minds to say that somewhere else it is different, I think that this is important at all levels."

Currently, there are compulsory courses, from the first year of university on, aimed at making students aware of different concepts of populations at risk, particularly immigrant patients.

One of the major problems noticed during interviews regarding the training course was the course's limited accessibility, particularly for emergency physicians because of their schedules and place of practice.

\section{Training tools}

Physicians noted their learning was improved by the use of videos. However, they also requested practical activities (problem solving), lectures in anthropology, and courses on geographic epidemiological profiles related to infectious diseases.

\section{Working material}

Participants noted an interest in receiving flyers for summarizing which administrative forms to fill out. They also expressed their need for an Internet page or an internet document to summarize key points and provide useful links. The lack of availability of medical interpreters, especially for unplanned visits (emergencies), was the main barrier to putting into practice their competency in trialogue. Finally, one group reported an interest in receiving patients' feedback on what they thought about the care they received.

\section{Quantitative dimension}

All 18 residents agreed to participate and answered the MAQ before (D-1), shortly after (D+3), and three months after $(\mathrm{D}+90)$ the training. Details of the studied population are reported in Table 2. Two residents were not able to complete one of the questions from the MAQ before the course, and one of them at D+3. Full data was therefore available for 16 residents. Sensitivity analysis shows that the worst case scenario does not change the study conclusion. Missing data therefore did not introduce any important bias.

Table 2. Profile of the 18 university outpatient clinic residents starting in spring $2010(\mathrm{~N}=18)$

\begin{tabular}{|c|c|c|}
\hline Variable & $\mathrm{n}$ & $\%$ \\
\hline \multicolumn{3}{|l|}{ Gender } \\
\hline Women & 11 & 61 \\
\hline Men & 7 & 39 \\
\hline Swiss nationality & 13 & 72 \\
\hline \multicolumn{3}{|l|}{ Ethnicity } \\
\hline Western & 15 & 83 \\
\hline African & 1 & 6 \\
\hline Latino & 2 & 11 \\
\hline \multicolumn{3}{|l|}{ Language (in addition to French) } \\
\hline English & 15 & 83 \\
\hline German & 12 & 66 \\
\hline Spanish & 7 & 39 \\
\hline Italian & 6 & 33 \\
\hline Other & 4 & 22 \\
\hline Speaks one additional language & 7 & 39 \\
\hline Speaks two additional languages & 8 & 44 \\
\hline Speaks three additional languages & 3 & 17 \\
\hline \multicolumn{3}{|l|}{ Education } \\
\hline Swiss Medicine Doctor (MD) & 13 & 72 \\
\hline Partly abroad & 14 & 78 \\
\hline Internal medicine & 3 & 17 \\
\hline General medicine & 15 & 83 \\
\hline \multicolumn{3}{|l|}{ Years of post-graduate education } \\
\hline $1-3$ yrs & 2 & 11 \\
\hline $4-6$ yrs & 13 & 72 \\
\hline$>6$ yrs & 3 & 17 \\
\hline
\end{tabular}

As shown in Table 3, three days after the course, the median difference (5.5 points) is significantly higher than zero $(\mathrm{df}=15, \mathrm{z}=2.4, \mathrm{p}=0.015)$, rising from a median of 33 to 38 points, showing an increase in the physicians' declared ability. At three months, the median difference was maintained at 4.5 points $(\mathrm{df}=15, \mathrm{z}=2.4, \mathrm{p}=0.018)$. At three months, median differences were mostly explained by an increase in the field of knowledge acquisition $(\Delta=4.5, \mathrm{df}=17$, $\mathrm{z}=3, \mathrm{p}=0.003$ ), and to a lesser degree by an increase in the field of skills $(\Delta=1, \mathrm{df}=15, \mathrm{z}=2.0, \mathrm{p}=0.05)$. As for attitudes, these did not seem to be affected by the course in the short 
term $(\Delta=0, \mathrm{df}=17, \mathrm{z}=0.7, \mathrm{p}=0.475)$ or the long term $(\Delta=0.5$, $\mathrm{df}=17, \mathrm{z}=1.3, \mathrm{p}=0.183)$.

\section{Discussion}

In this pre-post evaluation study of CCC training of 18 new residents in one of the four Swiss university outpatient clinics, we found that a training course of one-and-a-half hours focused on culture, trialogue and stereotypes has a clear impact on residents' declared cultural competency when measured three months after the initial training course, particularly in terms of knowledge, slightly in terms of skills, but not in terms of attitudes. This is encouraging given that our course was short compared to other courses in the literature that included up to 60 hours of training over weeks to months. ${ }^{15,16}$ The content was similar to some earlier published curricula though quite different from others. ${ }^{3,5}$ This is the first time that an evaluation of this kind of training course has been carried out at a local level and results match the literature fairly well.

In the area of knowledge, we show statistically a significant increase from three days to three months. This is in accord with results from the international literature: Beach's systematic review of CCC training ${ }^{15}$ shows that of the three areas of learning, the one which demonstrates the most noticeable improvement is knowledge. The results obtained by Culhane-Pera using the MAQ also prove such knowledge acquisition. $^{20}$

Attitudes were already very highly rated by residents' prior to the training. Residents are likely to over-assess themselves in terms of attitudes in general. More specifically, this group of resident physicians was already aware of the topic of immigrant populations and aspects of culture, given that they applied to study at the PMU and are mostly following the general medicine syllabus that integrates learning about diversity. Their scores remained stable after training and clinical practice. The very fact of practicing medicine brings with it a heightened awareness of multiculturalism also. ${ }^{15}$

Regarding the unexpected but interesting decrease in skill competencies after three months $(D+90)$ to levels lower than those recorded at D+3, several hypotheses can be considered. Given the high score obtained immediately after training, one can assume that primary-care residents were enthusiastic about their knowledge increase; enthusiasm which they correlated to their practical capacity, therefore over-assessing their skills. Then, when face-to-face with their real skill-levels with diverse, multicultural patients, they might have been better able to accurately assess themselves. A second reason for the decrease in skills could be related to the fact that the participants for whom a score decrease was observed in this area worked in the emergency department; one possible explanation being that in this department it is more difficult to focus on cultural differences due to the limited time available. These findings are in line with the literature - particularly an article mentioning that lack of time and communication problems are factors that favour stereotyping. ${ }^{23}$ Finally it is difficult to teach skills and show their impact with only one teaching session of one-and-a-half hours.

Our qualitative findings provided more detail on the impact of this kind of teaching and learning on a group of internal medicine residents with less than six years of postgraduate training. Our residents seem open-minded and enthusiastic that the training helped them cope with range of uncertainties related to the political status and everyday life of immigrant and forced migrant populations. It provided working guidelines for practice: working effectively with an interpreter, being aware of the patient's origin and politic status, and better understanding the impact of customs, religion or beliefs on treatment and disease. Physicians must be empowered to establish good communications with their patients if they want to understand cultural matters. The relevance of such training is even more important as it improves medical care, not only for patients of diverse origins but for Swiss patients as well. When residents work with immigrants they need to be excellent physicians, to take ethical principles into account, to integrate networking activities and to advocate for the patient: ${ }^{24}$ these four aspects are useful in the care of every patient. Some physicians reminded us that the Swiss population is unique, with its four languages and plethora of cultural differences, having either a German, French or Italian influence. Even people from urban areas, or those who live in more rural or mountainous regions, or come from neighbouring countries such as Germany, England or France, have noticeably different beliefs, disease portrayals and use of the healthcare system. CCC training not only brings awareness of multicultural issues but also helps participants understand their own cultures, perception of others and preconceived ideas.

However, some feedback concerning the context, and also the pedagogical method, needs to be addressed. The residents would like to have: more hours of training, including clinical practice, training at times convenient to their schedules and more support from chief residents to help them manage challenging cross-cultural situations, particularly in the emergency department where it is very difficult to take time with immigrant patients. Residents in general are also interested in specific themes such as geopolitics, epidemiology, religion and customs of less developed countries, as they relate to the patient-centred approach. While previous literature shows that cross-cultural competency training can lead to improvement in communication and adherence to therapy, ${ }^{25}$ a recent systematic review ${ }^{16}$ concluded that there is limited experimental research which shows a positive causal relationship between CCC training and improved patient outcomes. They proposed an algorithm to guide educators to demonstrate the impact on patient outcomes, and this should guide future research in this area. ${ }^{16}$ 
Table 3. Analysis of score differentials pre and post-cross-cultural education after three months of clinical practice

\begin{tabular}{|c|c|c|c|c|c|}
\hline \multirow{2}{*}{ Measure } & $\begin{array}{c}\text { Before } \\
\text { (reference) }\end{array}$ & $\begin{array}{c}\text { After } \\
3 \text { days }\end{array}$ & $\begin{array}{c}\text { After } \\
3 \text { months }\end{array}$ & $\Delta^{*}$ after 3 days & $\Delta$ after 3 months \\
\hline & Median & Median & Median & Median $\Delta\left(z, p\right.$ value $\left.^{\dagger}\right)$ & Median $\Delta(\mathrm{z}, \mathrm{p}$ value $)$ \\
\hline \multicolumn{6}{|l|}{ Knowledge $(n=18)$} \\
\hline $\begin{array}{l}\text { Definition of "culture" } \\
\text { Beliefs/values of healthcare } \\
\text { Practical situation } \\
\text { Negotiation } \\
\text { Specific actions } \\
\text { Anthropology } \\
\text { Knowledge score (max } 24 \text { pts) }\end{array}$ & $\begin{array}{c}2 \\
1 \\
1 \\
1 \\
0.5 \\
0 \\
7\end{array}$ & $\begin{array}{c}3 \\
2 \\
2 \\
2 \\
1.5 \\
1 \\
11\end{array}$ & $\begin{array}{c}2.5 \\
2 \\
2 \\
2 \\
1 \\
0 \\
11\end{array}$ & $\begin{array}{l}1(z=2.7, p=0.006) \\
1(z=2.0, p=0.046) \\
1(z=0.8, p=0.425) \\
1(z=3.4, p=0.001) \\
0(z=1.9, p=0.056) \\
0(z=2.6, p=0.009) \\
4(z=3.1, p=0.002)\end{array}$ & $\begin{array}{c}0(z=2.8, p=0.005) \\
1(z=2.1, p=0.037) \\
1(z=1.7, p=0.080) \\
1(z=2.5, p=0.011) \\
0(z=1.1, p=0.267) \\
0(z=1.7, p=0.081) \\
4.5(z=3.0, p=0.003)\end{array}$ \\
\hline \multicolumn{6}{|l|}{ Attitude $(n=18)$} \\
\hline $\begin{array}{l}\text { Own cultural awareness } \\
\text { Respect of other cultures } \\
\text { Impact of socio-cultural factors } \\
\text { Sensitivity to heterogeneity } \\
\text { Attitude score (max } 16 \text { pts) }\end{array}$ & $\begin{array}{c}3 \\
3 \\
3 \\
3 \\
12\end{array}$ & $\begin{array}{c}3 \\
3 \\
3 \\
3 \\
12\end{array}$ & $\begin{array}{c}3 \\
3 \\
3 \\
3.5 \\
13.5\end{array}$ & $\begin{array}{l}0(z=1.7, p=0.098) \\
0(z=0.4, p=0.670) \\
0(z=-0.5, p=0.616) \\
0(z=0.1, p=0.923) \\
0(z=0.7, p=0.475)\end{array}$ & $\begin{array}{l}0(z=1.3, p=0.189) \\
0(z=0.6, p=0.562) \\
0(z=1.1, p=0.256) \\
0(z=0.4, p=0.655) \\
0.5(z=1.3, p=0.183)\end{array}$ \\
\hline \multicolumn{6}{|l|}{ Skills } \\
\hline $\begin{array}{l}\text { Investigate culture }(n=18) \\
\text { Adapt anamnesis }(n=18) \\
\text { Respect status }(n=18) \\
\text { Negotiate diagnostic approach }(n=18) \\
\text { Use of interpreter }(n=17) \\
\text { Identity without stigmatization }(n=16) \\
\text { Skills score (max } 24 \text { pts, } n=16)\end{array}$ & $\begin{array}{c}2 \\
2 \\
2 \\
2 \\
2 \\
2 \\
14\end{array}$ & $\begin{array}{c}2 \\
3 \\
2 \\
2 \\
3 \\
3 \\
16\end{array}$ & $\begin{array}{c}2.5 \\
2.5 \\
3 \\
3 \\
3 \\
3 \\
16\end{array}$ & $\begin{array}{c}0(z=0.4, p=0.706) \\
0(z=1.3, p=0.187) \\
0(z=1.1, p=0.258) \\
0.5(z=1.8, p=0.074) \\
0(z=2.2, p=0.031) \\
1(z=2.6, p=0.010) \\
1.5(z=1.8, p=0.065)\end{array}$ & $\begin{array}{c}0(z=0.8, p=0.426) \\
0(z=0.3, p=0.763) \\
1(z=1.7, p=0.081) \\
0.5(z=2.5, p=0.011) \\
0(z=1.1, p=0.284) \\
1(z=1.7, p=0.086) \\
1(z=2.0, p=0.050)\end{array}$ \\
\hline Overall average score $(n=16)$ & 33 & 38 & 37.5 & $5.5(z=2.4, p=0.015)$ & $4.5(z=2.4, p=0.018)$ \\
\hline
\end{tabular}

${ }^{*} \Delta=$ difference,$\uparrow \mathrm{p}$ value $=$ probability of the null hypothesis been correct using Wilcoxon's signed rank test

\section{Limitations}

Despite the small size of this study, the qualitative evaluation encompassed all physicians who started their rounds at the PMU in April 2010. The small sample size of our study limits its power to detect smaller pre-post intervention changes. Also, our study did not include a control group due to logistical limitations. We therefore cannot assume a causal association between observed changes and the courses. Participants could also have been subject to social desirability bias. However, given that no changes were observed for skills, if this bias was present, it did not affect all questions. We also assumed the MAQ conserved its psychometric properties without having had the French version validated. The fact that the person who conducted the focus groups was one of the teachers of CCC could have influenced the opinions expressed by the residents. Otherwise, it was very useful to hear directly what expectations for the training course were. The study was done in a single institution thereby limiting its external validity. This is particularly true given the medical curriculum at PMU and the institution's social mission, making it likely that residents were sensitive to the topic of immigrant health even before the CCC training.

\section{Conclusions}

This study was the first to evaluate the impact of a CCC training program for internal medicine residents in Switzerland. While the program was brief, it appeared to have a positive impact on residents' knowledge of CCC and skills. Residents provided valuable perspectives on the CCC training experience, most of which were positive, but some provided critical insights and areas for improvement. These will help to shape our own training and to inform the field overall. Future educational research should include randomized, controlled trials comparing different interventions and measuring outcomes that move beyond self-report and include direct measures of communication and patientcentered care.

\section{Acknowledgments}

The authors would like to thank the 18 residents involved in this study, Mr. Orest Weber and Ms. Esther-Amélie Dieserens for their help and participation in the analysis of qualitative part, as well as Mr. Brooks for his precious help in correcting this English translation. No funding was necessary for this study as it was carried out as part of a student's masters' program, in association with Lausanne's PMU.

\section{Conflict of Interest}

The authors declare that they have no conflict of interest.

\section{References}

1. Althaus F, Hudelson P, Domenig D, Green AR, Bodenmann P. Compétences cliniques transculturelles et pratique médicale. Forum Med Suisse. 2010;10(4):79-83. 
2. Althaus F, Paroz S, Renteria SC, Rossi I, Gehri M, Bodenmann P. La santé des étrangers en Suisse: les médecins ont-ils mieux à faire ou peuvent-ils mieux faire? Forum Med Suisse. 2010;10(4):59-64.

3. Green AR, Betancourt JR. Cultural competence: a patient-based approach to caring for immigrants. In: Walker PF, Barnett ED, editors. Immigrant medicine. Boston: Sounders Elsevier; 2007:83-98.

4. Betancourt JR. Cross-cultural medical education: conceptual approaches and frameworks for evaluation. Acad Med. 2003;78(6):560-9.

5. Carrillo JE, Green AR, Betancourt JR. Cross-cultural primary care: a patient-based approach. Ann Intern Med. 1999;130(10):829-34.

6. Green AR, Betancourt JR, Carrillo JE. Integrating social factors into crosscultural medical education. Acad Med. 2002;77(3):193-7.

7. Park ER, Chun MB, Betancourt JR, Green AR, Weissman JS. Measuring residents' perceived preparedness and skilfulness to deliver cross-cultural care. J Gen Intern Med. 2009;24(9):1053-6.

8. Miller E, Green AR. Student reflections on learning cross-cultural skills through a 'cultural competence' OSCE. Med Teach. 2007;29(4):e76-84.

9. Greer JA, Park ER, Green AR, Betancourt JR, Weissman JS. Primary care resident perceived preparedness to deliver cross-cultural care: an examination of training and specialty differences. $J$ Gen Intern Med. 2007;22(8):1107-13.

10. Bigby JA. Beyond cultures: strategies for caring for patients from diverse racial, ethnic, and cultural groups. In: Bigby JA, editor. Cross-cultural medicine. Philadelphia: American College of Physicians; 2003:1-28.

11. Hudelson P. Contextualizing cultural competence training of residents: results of a formative research study in Geneva, Switzerland. Med Teach. 2006;28(5):465-71.

12. Kleinman A, Eisenberg L, Good B. Culture, illness, and care: clinical lessons from anthropologic and cross-cultural research. Ann Intern Med. 1978;88(2):251-8.

13. Grin G. Rôles et pratiques des interprètes et des médiateurs culturels: enquête anthropologique sur le réseau de soins de Lausanne. In: Guex P, Singy P, editors. Quand la médecine a besoin d'interprètes. Geneva:
Médecine et Hygiène; 2003:141-163.

14. Wilkinson R, Marmot M. Social determinants of health: the solid facts Geneva: World Health Organization; 2003.

15. Beach MC, Price EG, Gary TL, Robinson KA, Gozu A, Palacio A, et al. Cultural competence: a systematic review of health care provider educational interventions. Med Care. 2005;43(4):356-73.

16. Lie DA, Lee-Rey E, Gomez A, Bereknyei S, Braddock CH. Does cultural competency training of health professionals improve patient outcomes? A systematic review and proposed algorithm for future research. J Gen Intern Med. 2011;26(3):317-25.

17. Office Fédéral de la Statistique. Portrait démographique de la Suisse édition 2009. Neuchâtel: OFS; 2009.

18. Office Fédérale de la Santé Publique. Stratégie migration 2008-2013. Bern: OFSP; 2007:9.

19. Pope C, Mays N. Qualitative research in health care. Oxford: Blackwell Publishing; 1996.

20. Culhane-Pera KA, Reif C, Egli E, Baker NJ, Kassekert R. A curriculum for multicultural education in family medicine. Fam Med.1997;29(10):71923.

21. Crandall SJ, George G, Marion GS, Davis S. Applying theory to the design of cultural competency training for medical students: a case study. Acad Med. 2003;78(6):588-94.

22. Gozu A, Beach MC, Price EG, Gary TL, Robinson K, Palacio A, et al Self-administered instruments to measure cultural competence of health professionals: a systematic review. Teach Learn Med. 2007;19(2):180-90.

23. Durieux-Paillard S, Loutan L. Cultural diversity and stereotyping: implication for the medical practice. Rev Med Suisse. 2005;1(34):2208-13.

24. Bodenmann P, Gehri M. Soins aux migrants: une opportunité de pratiquer une meilleure médecine pour tous! Forum Med Suisse. 2010;10(4):57-8.

25. Paez KA, Allen JK, Beach MC, Carson KA, Cooper LA. Physician cultural competence and patient ratings of the patient-physician relationship. J Gen Intern Med. 2009;24(4):495-8. 